\title{
INCIDENCIA DE UN AMBIENTE DE APRENDIZAJE BLENDED, EN LA TRANSFORMACIÓN DE COMPETENCIAS MATEMÁTICAS EN ESTUDIANTES UNIVERSITARIOS
}

\author{
Impact of a blended learning environment \\ in the transformation of Math skills in university students
}

\author{
Andres Chiappe $^{1}$. Guillermo Antonio Manjarrés ${ }^{2}$
}

Resumen: El presente artículo describe una investigación que da cuenta de la incidencia de un Ambiente Virtual de Aprendizaje (AVA) en el fortalecimiento de algunas competencias matemáticas en estudiantes universitarios. La investigación permitió contrastar el estado de desarrollo de varias competencias matemáticas en estudiantes universitarios de primer semestre, antes y durante una experiencia de interacción en un Ambiente Virtual de Aprendizaje, como complemento de un curso presencial de Geometría. Los resultados obtenidos en la investigación permitieron identificar algunas transformaciones en los estudiantes, sobre todo en su capacidad de identificar, plantear y resolver problemas, en sus habilidades para comunicarse de manera oral y escrita y en su capacidad de aplicación de los conocimientos matemáticos al campo de la Ingeniería Civil.

Palabras clave: Ambiente virtual de aprendizaje. Competencias matemáticas. Geometría. Enseñanza de las matemáticas.

\begin{abstract}
This paper describes a research project that accounts for the impact of a Virtual Learning Environment (VLE) in strengthening some Math skills in university students. The research allowed us to compare the state of development of some mathematical skills in students of first semester, before and during an experience of interaction in a Virtual Learning Environment, in addition to a face to face course in Geometry. The research results helped identify some changes in students, especially in their abilities to identify and solve problems, communicate orally and in writing and in their ability to apply mathematical knowledge to the Civil Engineering field.
\end{abstract}

Keywords: Virtual learning environment. Blended learning. Math skills. Geometry. Mathematics education.

\footnotetext{
${ }^{1}$ Universidad de la Sabana (Unisabana), Bogotá, Colombia. Km 7 Autopista norte de Bogotá, Campus Universitario Puente del Común, Bogotá, Colombia. andres.chiappe@unisabana.edu.co

${ }^{2}$ Universidad de la Sabana (Unisabana), Bogotá, Colombia.
} 


\section{Introducción}

Las tecnologías de la información y comunicaciones (TIC) están provocando continuas transformaciones socio-económicas que han impactado enormemente el ámbito de lo educativo, generando posibilidades nunca antes vistas para el desarrollo de tareas organizativas, docentes y de investigación. (CARDONA, 2005).

Un escenario muy activo de integración de las TIC en la educación se caracteriza por la complementariedad entre lo presencial y lo virtual, denominado blended learning. Estos escenarios apoyados en las TIC

$[\ldots]$ permiten la interactividad continua y permanente entre los estudiantes y docentes con acceso a ellas, eliminando las barreras espacio temporales, haciendo posible la disposición de múltiples profesores para múltiples alumnos, la comunicación entre los propios alumnos, la creación de entornos de aprendizaje diversos [...]. (CABERO, 1995, p. 47).

En ese sentido, se configura un reto importante para las universidades con relación a la necesidad de innovar no sólo en tecnología que apoye estos procesos, sino también en las transformaciones de sus concepciones y prácticas pedagógicas. Desde luego, la actividad del docente universitario requiere un cambio en ciertas habilidades al menos en cuatro áreas fundamentales: pedagógica, social, organizativa y técnica (CHAUPART; CORREDOR; MARÍN, 1998). Por su parte, el estudiante deberá también cambiar tanto la forma de aproximarse al conocimiento (REYNOLDS, 2008), como sus hábitos de estudio (MUÑOZ, 2008).

El contexto en el cual se desenvolvió esta investigación incluyó un ejercicio de construcción de un AVA que respondió a las consideraciones anteriormente mencionadas y que se implementó bajo criterios que buscaron la generación de un proceso formativo activo, más autónomo, en el cual se pudieran proveer las condiciones necesarias para el fortalecimiento de competencias matemáticas en los estudiantes. Según la Asociación Nacional de Profesores de Matemáticas de Estados Unidos (NATIONAL COUNCIL OF TEACHERS OF MATHEMATICS, 2000, p. 24), los avances e inmersión de las TIC en el campo educativo permiten "aprender más matemáticas y en mayor profundidad con el uso apropiado de la tecnología", permitiendo grandes transformaciones en cuanto a las competencias matemáticas.

En la asignatura Geometría del programa de Ingeniería Civil de la Corporación Universitaria Minuto de Dios, se ha detectado desde hace ya varios años la dificultad que presentan los estudiantes para interpretar y analizar conceptos y elementos relacionados con el tema de las secciones cónicas, argumentar procedimientos y resultados sobre ejercicios propuestos, proponer algoritmos analíticos y geométricos en la resolución de problemas, relacionar dicho tema con la vida cotidiana, transferir los conocimientos estudiados a aplicaciones sencillas en el campo de la Ingeniería Civil y participar activamente en clase a través de la comunicación con el docente y con el grupo, bien sea en el aula o en horarios extraclase.

Esta situación reviste especial gravedad toda vez que la comprensión, manejo de los conceptos y el desarrollo de competencias matemáticas relacionadas con la Geometría son esenciales para el posterior desempeño de los estudiantes durante el resto de su proceso formativo como ingenieros. 
Incidencia de un ambiente de aprendizaje blended ...

Teniendo en cuenta esta problemática se trazó el proceso investigativo orientado sobre la siguiente pregunta: ¿De qué manera un Ambiente Virtual de Aprendizaje como complemento a las clases presenciales, incide en el desarrollo de competencias matemáticas en estudiantes de Ingeniería Civil?

\section{Acerca de los AVA como apoyo a la presencialidad}

El aprendizaje híbrido o blended learning ha sido definido de diversas formas y por múltiples autores. Sin embargo, se reconocen algunos elementos en común en la literatura especializada sobre el tema. Para efectos de esta investigación se reconoció el concepto como: "el término blended learning es usado para describir el aprendizaje que mezcla varios eventos basados en actividades educativas, incluyendo asistencia presencial en el salón de clase, aprendizaje a distancia (sincrónicos) y aprendizaje autónomo, entre otros." (VALIATHAN, 2002, p. 31 apud LOZANO; BURGOS, 2008, p. 130).

En ese orden de ideas, teniendo en cuenta los objetivos del aprendizaje, los contenidos y materiales de aprendizaje a utilizar, las actividades de aprendizaje, los medios de comunicación, el modelo institucional utilizado, las actividades del docente, entre otros, se han identificado los siguientes elementos dentro de los más frecuentemente utilizados en los ambientes de aprendizaje híbridos: instrucción presencial, instrucción interactiva, medios de comunicación, foros de discusión, contenidos para el aprendizaje autónomo, recursos, actividades tales como el envío de tareas y de evaluaciones. (ESCAMILLA, 2002).

\section{Fundamentación del diseño del AVA}

El ambiente de aprendizaje blended y los recursos educativos ubicados en su interior se diseñaron e implementaron con base en principios pedagógicos institucionales de la Corporación Universitaria Minuto de Dios.

Teniendo en cuenta las características de los estudiantes y la orientación pedagógica institucional de esta Universidad, se optó por fundamentar el diseño del AVA desde un modelo educativo praxeológico cuyo eje central es la formación integral del educando, sobre el cual se procura un desarrollo integral de todas las dimensiones del ser humano. En este orden de ideas, el estudiante debe relacionar el saber (teoría) con la práctica (praxis) y disponer de aptitudes para integrar su proyecto de vida y laboral en el contexto social actual.

Para el caso de esta investigación, cuyo eje central temático fue el estudio de las secciones cónicas en el campo bidimensional, se dispuso de una serie de momentos ordenados y mediados a través de las TIC, como herramientas didácticas que permitieron integrar el pensamiento y la acción, los valores e intenciones y el sentido y la eficacia. (JULIAO, 2002). El proceso didáctico partió de la práctica (ver) permitiendo la toma de decisiones, las cuales pudieron ser constatadas (juzgar) con la ayuda de procesos de razonamiento inductivo, los cuales permitieron comprobar su veracidad y rigurosidad en la formulación, planeación y elaboración estratégica de la acción (actuar). Se obtuvo entonces una propuesta que partió de una práctica realizada en el aula de clase que finalmente permitió llegar a la conceptualización de las secciones cónicas (devolución creativa). De esta manera, los estudiantes pudieron desarro- 
Chiappe, A.; Manjarrés, G. A.

llar la capacidad de presentar argumentos matemáticos acerca de las relaciones geométricas, además de utilizar la visualización, el razonamiento espacial y la modelación geométrica para resolver problemas.

\section{Desarrollo del proyecto}

El proyecto se diseñó y llevo a cabo como una experiencia investigativa de naturaleza cualitativa, a manera de estudio de caso, en el cual se utilizaron técnicas de análisis tanto cualitativas como cuantitativas, que permitieron abordar de una manera más amplia las transformaciones ocurridas en las competencias matemáticas de los estudiantes en el marco de la interacción en un Ambiente Virtual de Aprendizaje, como apoyo a las clases presenciales de la asignatura Geometría.

Las competencias matemáticas asociadas al aprendizaje del tema "Secciones cónicas bidimensionales" y que fueron la base para la formulación posterior de las categorías de análisis de la investigación se plantearon como:

- Capacidad para identificar, plantear y resolver problemas.

- Capacidad de comunicación oral y escrita.

- Aplicación de conocimientos de Ciencias Básicas y Ciencias de la Ingeniería Civil.

Pese a que se pudiera advertir que estas competencias tienen una connotación general, se les reconoce como competencias matemáticas tal y como se han definido en el "Proyecto Tuning América Latina" para el Área de Matemáticas. El "Proyecto Tuning América Latina” es relevante para la investigación en tanto se reconoce como un referente internacional en el tema de competencias y es tenido en cuenta tanto en los procesos de diseño curricular de los Programas de Ingeniería Civil, como en los procesos institucionales de acreditación de alta calidad.

Participaron en esta experiencia investigativa veinte estudiantes de primer semestre de la jornada diurna de Ingeniería Civil, quienes tomaron la asignatura Geometría. A dicho grupo de estudiantes se les hizo un seguimiento y observación a su desempeño y participación en dicha asignatura durante todo el semestre lectivo haciendo énfasis en dos momentos diferentes: antes y durante la utilización del Ambiente Virtual de Aprendizaje.

El proceso investigativo se llevó a cabo de la siguiente manera:

Todo el grupo de estudiantes asistió normalmente a sus clases presenciales durante todo el semestre e igualmente participaron de las interacciones propuestas dentro del AVA, el cual tuvo una duración de cinco semanas y se implementó en la plataforma Moodle.

En el AVA se incluyó un Objeto de Aprendizaje (OA), entendido éste como un material educativo digital reutilizable (CHIAPPE, 2008). Dicho OA se diseñó con el ánimo de ayudar a que los estudiantes desarrollaran su capacidad matemática, ofreciendo experiencias que estimularan su curiosidad y confianza. Además ayudó a realizar actividades que promovieran la participación activa de los estudiantes, induciendo a la aplicación de las matemáticas en situaciones reales y la comprensión y uso de patrones y relaciones como parte de la competencia matemática. Finalmente el $\mathrm{OA}$ ayudó a propiciar oportunidades para usar adecuadamente el lenguaje matemático con el fin de comunicar adecuadamente ideas matemáticas y ofrecer experiencias en las que los estudiantes pudieran explicar, justificar y refinar su propio pensamiento, sin limitarse a repetir lo que dice un libro de texto. 
Incidencia de un ambiente de aprendizaje blended ...

A lo largo del semestre se consignó periódicamente en un diario de campo la forma como los estudiantes resolvían problemas de manera que se pudieran evidenciar transformaciones en este aspecto asociadas a la interacción con el AVA.

Se realizó un análisis de contenido a la documentación consignada en el AVA que mostraba la producción escrita y oral de los estudiantes durante todo el semestre con el fin de identificar las transformaciones en dicha producción. Las fuentes de información para este proceso fueron los trabajos, las pruebas escritas y la participación en clase. Durante la interacción en el AVA se tuvieron además las siguientes fuentes adicionales: chats, foros, audios, ejercicios orales, ejercicios escritos, y las evaluaciones o pruebas escritas.

Mediante una prueba diagnóstica o inicial, se identificó el nivel de desarrollo de las competencias interpretativas, argumentativas y comunicativas existentes en los estudiantes sobre el tema de las secciones cónicas bidimensionales. Dichas competencias fueron el insumo principal de las categorías de análisis anteriormente formuladas.

Se llevó a cabo el seguimiento individual para cada estudiante con el fin de identificar transformaciones en las competencias matemáticas desde lo personal y desde la dimensión grupal. En ese sentido, con relación a la competencia capacidad para identificar, plantear y resolver problemas, se abordaron los siguientes aspectos a manera de categorías de análisis: . Su capacidad para hacer un correcto seguimiento de los procedimientos matemáticos. - El manejo adecuado del lenguaje matemático.

. Su capacidad para llevar a cabo procesos de jerarquización de operaciones.

Por otra parte, con relación a la competencia capacidad de comunicación oral y escrita se abordaron, los siguientes aspectos a manera de categorías de análisis:

. La capacidad del estudiante para expresarse de manera escrita.

- La coherencia del discurso del estudiante con la acción que éste ejecuta.

. La capacidad de síntesis por parte del estudiante.

Finalmente, con relación a la competencia aplicar conocimientos de Ciencias Básicas y Ciencias de la Ingeniería Civil, se abordaron los siguientes aspectos a manera de categorías de análisis:

. La claridad conceptual expresada por el estudiante.

- La habilidad del estudiante para llevar a cabo procesos de graficación.

- La coherencia manifestada por el estudiante entre la acción y la aplicación.

El proceso de análisis e interpretación de la información recolectada se llevó a cabo de la siguiente manera:

La información recolectada antes y durante la experiencia de interacción en el AVA se clasificó en tablas las cuales permitieron hacer una aproximación a las fuentes de información generadas por cada estudiante. Estas tablas incluyeron información relevante para cada categoría de análisis y para cada competencia matemática. Luego de clasificada la información, se definieron las unidades de análisis de contenido, las cuales se extrajeron de la fuente original y se codificaron según la fuente, el momento en el cual se produjo la información, la categoría de análisis con la que se relacionaba y la competencia a la cual apuntaba. Cada unidad de análisis se consideró como un producto comunicativo en particular expresado por cada uno los estudiantes participantes. "Algunos análisis se centran en las inferencias que se realizan a partir del estudio de un tipo de productos comunicativos sobre otros productos comunicativos [...]." (PIÑUEL, 2002, p. 9). 
Los procedimientos de análisis cualitativos y cuantitativos se realizaron de forma conjunta y articulada de manera que fuera posible tener una mirada complementaria de la misma información. En ese orden de ideas, la cuantificación y procesamiento estadístico básico de los segmentos de texto extraídos de cada producto realizado por los estudiantes se contrastó con la interpretación cualitativa de dichos segmentos de texto.

La permanente actualización y comparación de estas tablas permitió proseguir con el proceso de interpretación cualitativa de la información, en la cual se pudo hacer una aproximación, no solo a la forma en que se desarrolló la experiencia investigativa sino hacia el reconocimiento e identificación de aquellas transformaciones relacionadas con las competencias matemáticas de los estudiantes que participaron en la investigación.

\section{Resultados y discusión}

En este apartado se presentan los principales hallazgos de este estudio como resultado de la interpretación de la información encontrada con relación a cada una de las competencias propuestas. Algunos de estos resultados apuntan a identificar ciertos aspectos en los cuales la incorporación de las TIC genera valor al aprendizaje y otros en los cuales puede presentar inconvenientes importantes que se deberán atender y prever.

Como producto del seguimiento y observación permanente de las actividades de los estudiantes y teniendo en cuenta el análisis realizado de la información generada por ellos, se encontró lo siguiente:

En cuanto a la primera competencia denominada capacidad para identificar, plantear y resolver problemas:

. De acuerdo a los trabajos escritos presentados por los estudiantes, no se observa ninguna transformación evidente en esta competencia. Tanto antes como durante la interacción en el AVA se presentaron trabajos escritos realizando un correcto seguimiento de los procedimientos matemáticos, utilizando un adecuado lenguaje matemático y demostrando la capacidad para realizar procesos de jerarquización de operaciones.

- Ante la ausencia casi total de participación activa de los estudiantes al inicio del semestre en las clases presenciales, se notó un aumento significativo de la participación de estudiantes durante la interacción en el AVA, en donde más de la mitad del curso participó activamente y de forma clara en los foros, a través de los audios y en los ejercicios orales. Lo anterior evidenció el fortalecimiento de su capacidad para describir en forma acertada el procedimiento para realizar un ejercicio, el aumento de la coherencia del discurso y la acción y el uso correcto del lenguaje matemático. Además, jerarquizaron adecuadamente las operaciones matemáticas en las propuestas de solución a los ejercicios.

. Las pruebas escritas realizadas por los estudiantes mostraron un aumento de más del 20\% en el uso correcto del simbolismo matemático durante la interacción en el AVA. Este adecuado manejo del simbolismo matemático representó un aumento en la capacidad de jerarquizar correctamente las operaciones matemáticas, definiendo correctamente un problema y resolviendo correctamente el manejo de algoritmos de solución. 
Incidencia de un ambiente de aprendizaje blended ... y escrita:

En cuanto a la segunda competencia denominada capacidad de comunicación oral

. Durante la interacción en el AVA se encontró una disminución del 10\% en el número de trabajos escritos presentados por los estudiantes en los cuales hubiera evidencia de una relación alta de coherencia del discurso con la acción. Lo anterior se expresa por lo general, al realizar los ejercicios de una manera sintética. En este último aspecto, se notó que los estudiantes resolvieron los ejercicios pero no presentaron resultados de manera sintetizada. La disminución de resultados positivos durante la interacción en el AVA, con relación a la capacidad de expresión escrita se debió a inconvenientes con el manejo de las TIC, sobre todo con relación al uso del editor de ecuaciones.

. Las actividades desarrolladas a través de los chat, los foros, los audios y los ejercicios orales mostraron un aumento significativo en la capacidad de expresión efectiva, oral y escrita. La comunicación generada en dichas herramientas mostró un nivel alto de coherencia del discurso con la acción y de síntesis argumentativa de los ejercicios propuestos.

. Durante la interacción en el AVA hubo un aumento de más del $45 \%$ en los estudiantes que evidenciaron mejoramiento tanto en redacción como en ortografía de las pruebas escritas (evaluaciones).

. Las evaluaciones escritas presentadas por los estudiantes durante la interacción en el AVA fueron más precisas, presentaron un desarrollo lógico con un adecuado simbolismo matemático conducente a una correcta solución a los ejercicios propuestos en estas evaluaciones. Además, en estas pruebas escritas los estudiantes presentaron las soluciones a manera de síntesis, lo cual no se evidenció en la etapa anterior.

En cuanto a la tercera competencia denominada aplicar conocimientos de Ciencias Básicas y Ciencias de la Ingeniería Civil:

. En los trabajos escritos producidos por los estudiantes no se logra evidenciar ninguna transformación relevante con relación a la aplicación de conocimientos. Los estudiantes presentaron durante todo el semestre un nivel similar de claridad conceptual al sugerir algoritmos lógicos matemáticos en la solución de problemas propuestos. Sin embargo, se notó que al interactuar en el AVA los estudiantes describieron en forma más precisa y oportuna procedimientos matemáticos basados en la claridad conceptual y lograron hacer mejores diseños de graficación para la aplicación de los conceptos adquiridos en Ciencias Básicas en situaciones propias del campo de la Ingeniería Civil.

. De la mano del aumento significativo de la participación activa de los estudiantes, se evidenció un aumento en la capacidad de expresarse de manera adecuada, sobre todo en los foros en donde se notó alta claridad conceptual en sus apreciaciones, presentaron gráficos en los foros que así lo requerían y dieron soluciones a ejercicios de aplicaciones inmediatas en las ciencias de la Ingeniería Civil.

Las transformaciones identificadas anteriormente se enfocan principalmente en cuanto al dominio de la claridad conceptual y la representación gráfica de los ejercicios propuestos en las evaluaciones escritas. 


\section{Conclusiones}

Los resultados encontrados suponen reconocer la complejidad propia del proceso de aprendizaje de la Geometría y evidencian sin duda las dificultades que los estudiantes presentan al respecto, lo cual confirma el diagnóstico institucional en esta materia.

Sin embargo, la experiencia de diseño e implementación de un AVA como parte de un proceso formativo basado en un esquema blended learning dejó un conjunto de aprendizajes institucionales y personales para los participantes de esta experiencia que bien vale la pena hacer explícitos:

- Debido a las características del AVA implementado, se permitió a los estudiantes expresarse de manera más natural y espontánea, en comparación con las clases presenciales, rompiendo la barrera entre estudiante-estudiante y entre estudiante-docente. Esto, generó la disminución del temor para expresarse; las intervenciones o participaciones fueron más concretas, precisaron sus consultas y propusieron secuencialmente soluciones inmediatas a los ejercicios propuestos. El aumento de los escenarios que permitieron un mejoramiento en la comunicación fue un factor decisivo para las transformaciones observadas durante esta experiencia.

. En las pruebas escritas se evidenció una gran similitud en los procedimientos matemáticos utilizados con aquellos procedimientos planteados en ejercicios ya resueltos a manera de ejemplos. Lo anterior dio pie para pensar que los estudiantes requieren de ciertos patrones iniciales para adquirir habilidades en el planteamiento y utilización de procedimientos matemáticos adecuados en la solución de los problemas propuestos. Esto puede llegar a convertirse en un lineamiento para el diseño de materiales educativos o de ambientes virtuales enfocados al aprendizaje de las matemáticas.

- Buena parte del éxito de los procesos formativos virtuales va de la mano de la participación activa de los estudiantes. Entendiendo que pueden existir algunos factores externos o personales que pudieran haber intervenido de alguna manera en los resultados de la investigación, se reconoce que el aumento de las capacidades de expresión de los estudiantes así como de las otras competencias matemáticas es consecuencia, en gran parte, del compromiso del estudiante con su propio proceso formativo. En ese orden de ideas, un AVA como el implementado en este proyecto, proporcionó los espacios, herramientas y actividades necesarias para propiciar un cambio mayor en la participación de los estudiantes evitando con esto la exaltación del rol de receptor pasivo de información.

- La producción oral del estudiante sin la utilización del ambiente virtual, es casi nula debido a la poca participación en clase, al poco tiempo disponible en las clases presenciales, o a la timidez del estudiante. Estos inconvenientes se sobrepasan debido a la naturaleza flexible de la interacción y disponibilidad de espacios de participación presentes en el AVA. Igualmente, la producción oral se fortalece en los estudiantes cuando utilizan el ambiente virtual de aprendizaje debido a que disponen de más oportunidades y tiempo a través de los foros, chats y otros recursos tecnológicos como la telefonía, lo cual incidió en el mejoramiento de la capacidad de comunicación con el docente o tutor y con sus compañeros de clase.

. La realimentación obtenida de los materiales implementados en el AVA es superior a la obtenida de los escasos materiales utilizados en el tablero durante el desarrollo de las clases presenciales. Esto puede considerarse como un factor no relacionado con la información obtenida en la investigación, pero que bien puede tener una incidencia importante, sobre todo 
Incidencia de un ambiente de aprendizaje blended ...

para la competencia aplicar conocimientos de ciencias básicas y ciencias de la ingeniería civil.

Finalmente, la utilización de un ambiente virtual de aprendizaje, como apoyo a las clases presenciales, proporciona un espacio facilitador de los procesos de enseñanza y aprendizaje, con el fin de generar cambios en las competencias matemáticas, favoreciendo la interpretación, adquisición y utilización de los conocimientos matemáticos, los cuales se evidenciaron en las distintas fuentes de información utilizadas en la presente investigación. Estas fuentes de información presentaron una gama de oportunidades para continuar con diversos procesos investigativos conducentes a contrastar e identificar otras competencias matemáticas en los estudiantes universitarios.

\section{Referencias}

CABERO, J. M. Nuevos canales de comunicación en enseñanza. Madrid: Centro de Estudios Ramón Areces, 1995.

CARDONA, G. Tendencias educativas para el siglo XXI: educación virtual online y @learning - elementos para la discusión. 2005. Tesis (PhD en Ciencias Pedagógicas) Universidad Javeriana, Bogotá, 2005. Recuperado de: <http://edutec.rediris.es/Revelec2/ revelec15/cardona.pdf>. Acceso en: 01 dic. 2009.

CHAUPART, J. M.; CORREDOR, M. V.; MARÍN, J. I. El tutor, el estudiante y su nuevo rol. In: --____ Desarrollo de ambientes de aprendizaje en educación a distancia. México: Universidad de Guadalajara, 1998. p. 97-109.

CHIAPPE, A. Materiales educativos digitales. In: SEMINARIO DE LA MAESTRIA EN INFORMÁTICA EDUCATIVA. Bogotá: Universidad de la Sabana, 2008.

ESCAMILLA, J. Selección y uso de tecnología educativa. México: Trillas, 2002.

JULIAO, C. La praxeología: una teoría de la práctica. Bogotá: Corporación Universitaria Minuto de Dios, 2002.

LOZANO, R.; BURGOS, V. Tecnología educativa en un modelo de educación a distancia centrado en la persona. México: Limusa, 2008.

MUÑOZ, G. Potencialidad pedagógica de las tecnologías interactivas en los mundos de vida de los "nativos digitales". In: CONGRESO INTERNACIONAL DE PEGAGOGÍA E INFANCIA, 2., 2008, Bogotá. Memorias... Bogotá: Universidad de la Sabana, 2008.

NATIONAL COUNCIL OF TEACHERS OF MATHEMATICS. Principles and standards for school Mathematics. Reston: NCTM, 2000.

PIÑUEL, J. Epistemología, metodología y técnicas del análisis de contenido. Madrid: Universidad Complutense, 2002.

REYNOLDS, J. Educar con tecnología hoy. [conferencia]. In: CONGRESO

INTERNACIONAL DE PEGAGOGÍA E INFANCIA, 2., 2008, Bogotá. Memorias... Bogotá: Universidad de la Sabana, 2008. 
Chiappe, A.; Manjarrés, G. A.

Artigo recebido em 03/03/12. Aceito em 29/10/12. 